\title{
Quantitative and functional analysis of PDC-E2-specific autoreactive cytotoxic $T$ lymphocytes in primary biliary cirrhosis
}

\author{
Hiroto Kita, ${ }^{1}$ Shuji Matsumura, ${ }^{1}$ Xiao-Song He, ${ }^{1}$ Aftab A. Ansari, ${ }^{2}$ Zhe-Xiong Lian, ${ }^{1}$ \\ Judy Van de Water, ${ }^{1}$ Ross L. Coppel, ${ }^{3}$ Marshall M. Kaplan, ${ }^{4}$ and M. Eric Gershwin ${ }^{1}$ \\ ${ }^{1}$ Division of Rheumatology, Allergy and Clinical Immunology, University of California at Davis School of Medicine, \\ Davis, California, USA \\ ${ }^{2}$ Department of Pathology, Emory University School of Medicine, Atlanta, Georgia, USA \\ ${ }^{3}$ Department of Microbiology, Monash University, Clayton, Victoria, Australia \\ ${ }^{4}$ Division of Gastroenterology, Tufts-New England Medical Center, Boston, Massachusetts, USA
}

Address correspondence to: M. Eric Gershwin, Division of Rheumatology, Allergy and Clinical Immunology, University of California at Davis School of Medicine, TB 192, One Shields Avenue, Davis, California 95616, USA. Phone: (530) 752-2884; Fax: (530) 752-4669; E-mail: megershwin@ucdavis.edu.

Received for publication November 26, 2001, and accepted in revised form March 25, 2002.

\begin{abstract}
While the pathologic mechanisms responsible for organ-specific tissue damage in primary biliary cirrhosis (PBC) remain an enigma, it has been suggested that the pathology is mediated by autoreactive $T$ cells infiltrating the intrahepatic bile ducts. Previously, we have documented that there is 100 -fold enrichment in the frequency of $\mathrm{CD}^{+}$autoreactive $\mathrm{T}$ cells in the liver that are specific for peptides encoded by the E2 components of the pyruvate dehydrogenase complexes (PDC-E2). We have also recently characterized the first MHC class I-restricted epitope for PDC-E2, namely amino acid 159-167, a region very similar to the epitope recognized by MHC class II-restricted CD4 $4^{+}$cells and by

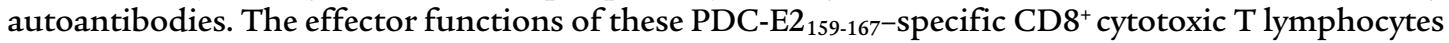
(CTLs) are not well understood. We have taken advantage of tetramer technology and report herein that there is tenfold increase in the frequency of PDC-E2 ${ }_{159-167}$-specific CTLs in the liver as compared with the blood in PBC. In addition, the precursor frequency of the CTLs in blood was significantly higher in early-stage PBC. Of interest was the fact that, upon stimulation with the peptide, the response of PDC-E2 ${ }_{159-167}$ tetramer-positive cells is heterogeneous with respect to IFN- $\gamma$ synthesis. These data, we believe for the first time, document the enrichment of autoantigen-specific $\mathrm{CD}^{+} \mathrm{T}$ cells in the $\mathrm{PBC}$ liver, suggesting that $\mathrm{CD8}^{+} \mathrm{T}$ cells play a significant role in the immunopathogenesis of $\mathrm{PBC}$.
\end{abstract}

J. Clin. Invest. 109:1231-1240 (2002). DOI:10.1172/JCI200214698.

\section{Introduction}

Primary biliary cirrhosis (PBC) is an autoimmune cholestatic liver disease characterized by the presence of antimitochondrial Ab's (AMAs) and intense biliary inflammatory response. The major mitochondrial antigen recognized by AMAs has been defined as the $\mathrm{E} 2$ component of the pyruvate dehydrogenase complex (PDC-E2) (1). Several lines of evidence suggest that $\mathrm{T}$ cells are implicated in the pathogenesis of PBC (2). This is exemplified by the presence of $\mathrm{CD} 4^{+}$and $\mathrm{CD}^{+} \mathrm{T}$ cell infiltrates within the portal tracts of the liver in PBC patients (3). MHC class II-restricted autoreactive $\mathrm{CD} 4^{+} \mathrm{T}$ cells specific for PDC-E2 have been identified from both peripheral blood and liver and the immunodominant target epitope has been defined to be amino acids 163-176 of PDC-E2 $(4,5)$. Moreover, we have recently identified an MHC class I-restricted CD8 ${ }^{+}$epitope of PDC-E2. This peptide, amino acid 159-167 of PDC-E2, can specifically induce CD8 ${ }^{+}$ cytotoxic T lymphocyte (CTL) lines from the PBMCs of HLA-A ${ }^{*} 0201^{+}$patients with PBC (6). These data suggest that much of the $T$ cell and the autoantibody responses in this disease are directed against PDC-E2.
Although autoreactive $\mathrm{CD}^{+} \mathrm{T}$ cells are thought to be involved in the pathogenesis of several autoimmune diseases (7-9), the role of PDC-E2-specific CD8 ${ }^{+} \mathrm{T}$ cells in the pathogenesis of $\mathrm{PBC}$ remains elusive. Previous investigations have demonstrated the accumulation of antigen-reactive $T$ cells at the site of the inflammation in several human autoimmune diseases as well as in murine models of human autoimmune diseases (9-12). It was thus reasoned that a comparative analysis of the precursor frequencies of PDC-E2-specific T cells isolated from the peripheral blood and from the liver of PBC patients would provide important information as to the relevance of such $T$ cells in the pathogenesis of disease. In a previous report from our laboratory, 100fold increase in the precursor frequency of PDC$\mathrm{E} 2$-specific $\mathrm{CD}^{+} \mathrm{T}$ cells in the hilar lymph nodes and liver compared with PBMCs from patients with $\mathrm{PBC}$ has been documented (13). The recent advances in the identification of antigen-specific $\mathrm{T}$ cells with peptideMHC tetramers, coupled with our identification of a HLA-A*0201-restricted immunodominant MHC class I epitope in PDC-E2, prompted us to use this technique 
and knowledge to determine whether there is a similar selective enrichment of autoreactive MHC class I-restricted $\mathrm{CD}^{+} \mathrm{CTL}$ in the diseased liver.

\section{Materials}

Patients and cells. The present study involved blood from $18 \mathrm{HLA}^{*} \mathrm{~A}^{*} 0201^{+}$patients with PBC (PBC 1 to PBC 18; four at stage I, five at stage II, four at stage III, and five at stage IV), four HLA-A* $0201^{+}$healthy controls (normal 1 to 4), and four HLA-A* $0201^{+}$patients with other chronic liver diseases (chronic liver disease 1 to 4 ; three alcoholic hepatitis and one granulomatous hepatitis), and four HLA-A2- ${ }^{-}$patients with PBC (A2 negative PBC 25 to 28). The HLA-A*0201 haplotype of the subjects was determined initially by using HLA-A2-specific mAbs MA2.1 and BB7.2 as described previously (14) and confirmed by standard molecular MHC class I typing. PBMCs were purified from venous blood using standard Ficoll-Histopaque gradient centrifugation techniques. Liver-infiltrating lymphocytes (LILs) were harvested from explanted liver tissue of six HLA-A*0201+ PBC patients undergoing liver transplantation (PBC 19 to PBC 24), as described (13). LILs were also harvested from excess liver tissue of two HLA-A* $0201^{+}$normal adult livers donated for transplantation (normal 5 to 6). The PBMCs and LILs were cryopreserved in 10\% DMSO/90\% FCS and stored in liquid nitrogen until used.

Synthetic peptides. A synthetic peptide corresponding to the previously defined immunodominant HLA$A^{*} 0201$-restricted CTL epitope of the autoantigen, amino acid 159-167 of the PDC-E2 (PDC-E2 $159-167$ ), was prepared (6). A previously defined HLA-A*0201-restricted CTL epitope, amino acid 18-27 of the hepatitis $B$ virus core protein $\left(\mathrm{HBc}_{18-27}\right)(15)$, and amino acid 58-66 of influenza matrix protein $\left(\right.$ Flu $\left.\mathrm{MP}_{58-66}\right)$ (16) were also used as negative controls. All peptides were synthesized with a free $\mathrm{NH}_{2}$ and a free $\mathrm{COOH}$ terminus and their purity confirmed by HPLC. Lyophilized peptides were reconstituted at $10 \mathrm{mg} / \mathrm{ml}$ in DMSO and diluted in the appropriate medium before use.

Synthesis of peptide-MHC tetramers. PDC-E2 ${ }_{159-167-H L A}$ A2 tetramers were generated as described (17). In brief, recombinant HLA-A*0201 with a 15 -amino acid substrate peptide for BirA-dependent biotinylation at its $\mathrm{C}$ terminus was expressed in Escherichia coli BL21(DE3)pLysS (Promega Corp., Madison, Wisconsin, USA) carrying pET-HLA-A*0201 plasmid (Promega Corp.) and isolated from inclusion bodies. Recombinant $\beta 2$ microglobulin was also expressed in the same way using a plasmid carrying $\beta 2$ microglobulin ( $\mathrm{pHN} 1-$ $\beta 2 \mathrm{~m}$ ), which was a gift from D.C. Wiley (Harvard University, Cambridge, Massachusetts, USA). The inclusion bodies were purified and dissolved in a urea-denaturing buffer. The monomeric MHC-peptide complexes were formed by combining the recombinant HLA-A*0201, recombinant $\beta 2$-microglobulin, and the nonapeptide PDC-E2 $2_{159-167}$ in an arginine-folding buffer. HLA$A^{*} 0201$ was folded in the presence of $\beta 2$-microglobulin and a specific peptide to form a peptide-MHC complex, following the procedure of Garboczi et al. (16). The complex was purified using a Sephacryl S100 column (Pharmacia Biotech Inc., Piscataway, New Jersey, USA). The MHC-peptide complexes were biotinylated enzymatically with BirA enzyme (Avidity, Denver, Colorado, USA), mixed with phycoerythrin-labeled Extraavidin (Sigma Chemical Co., Saint Louis, Missouri, USA) at a molar ratio of 4:1 to form the tetrameric peptide-MHC complex. Similarly prepared Flu $\mathrm{MP}_{58-66}$-HLA-A*0201 tetramers were used as controls.

Generation of peptide-induced CTL lines. Peptide-specific CTL lines were generated as described previously (6). Dendritic cells (DCs) were generated from PBMCs of HLA-A*0201-positive donors as described (18) and used as antigen-presenting cells (APCs). In brief, PBMCs were resuspended in RPMI-1640 (Life Technologies Inc., Grand Island, New York, USA) supplemented with 10\% heat-inactivated FCS, penicillin $(50 \mathrm{U} / \mathrm{ml})$, and streptomycin $(50 \mu \mathrm{g} / \mathrm{ml})$ (FCS medium), seeded in a $250-\mathrm{ml}$ culture flask, and incubated at $37^{\circ} \mathrm{C}$ for 1.5 hours. After removing nonadherent cells in the medium, the adherent cells were fed with FCS medium supplemented with GM-CSF (1,000 U/ml) (PreproTech Inc., Rocky Hill, New Jersey, USA) and IL-4 (1,000 U/ml) (PreproTech Inc.) and cultured at $37^{\circ} \mathrm{C}$. On day 7 , cells were harvested and incubated overnight in FCS medium containing PDC-E2 159-167 peptide $(10 \mu \mathrm{M})$. The cells were then washed and $\gamma$ irradiated (5,000 gy). Cryopreserved PBMCs or LILs were thawed, washed twice with HBSS, resuspended in FCS medium, and seeded in 24-well plates at $2 \times 10^{6}$ cells per well along with $2 \times 10^{5}$ peptidepulsed APCs. Cells were cultured at $37^{\circ} \mathrm{C}$ for a total of 14 days, with appropriate feeding of the cultures with media. On day three, recombinant human IL-2 (PeproTech) was added to each well at a final concentration of $10 \mathrm{U} / \mathrm{ml}$. In select experiments, PBMCs or LILs were labeled with 5-carboxyfluorescein diacetate succinimidyl ester (CFSE; Molecular Probes Inc., Eugene, Oregon, USA) prior to coculture with APCs. In these experiments the cryopreserved cells were thawed, washed twice with serum-free PBS, and labeled with $0.5 \mu \mathrm{M}$ CFSE for 10 minutes at $37^{\circ} \mathrm{C}$. The labeling was terminated by the addition of FCS, and subsequently the cells were washed six times with FCS medium. The CFSEstained cells were seeded in 24-well plates at $2 \times 10^{6}$ cells per well, along with $2 \times 10^{5}$ peptide-pulsed APCs, and cultured for 14 days as described above.

Flow-cytometric analysis and cell sorting. Peptide-specific CTL lines were prepared as described above, resuspended in $50 \mu \mathrm{l}$ of FACS buffer $(0.5 \%$ BSA and $0.05 \%$ sodium azide in $\mathrm{PBS}$ ), and incubated at $4{ }^{\circ} \mathrm{C}$ with $\mathrm{Fc} \gamma$ receptor $\mathrm{Ab}$ (Miltenyi Biotec, Auburn, California, USA) and NeutrAvidin (Molecular Probes Inc.) for $15 \mathrm{~min}-$ utes. Cells were stained with the appropriate peptideloaded PE-labeled HLA-A*0201 tetramer for $30 \mathrm{~min}$ utes at room temperature in the dark, washed, and subsequently incubated with FITC-labeled anti-CD8 Ab's (Caltag Laboratories Inc., Burlingame, California, USA) and tri-color (TC)-labeled anti-CD4 Ab's (Caltag 
Laboratories Inc.) for 30 minutes at $4{ }^{\circ} \mathrm{C}$ in the dark. After incubation, the cells were washed twice with FACS buffer, fixed with $1 \%$ paraformaldehyde in PBS, and analyzed by three-color flow cytometry using a FACScan (Becton Dickinson Immunocytometry Systems, San Jose, California, USA). The acquired data were analyzed with CELLQUEST software (BD Biosciences, San Jose, California, USA). In some experiments, both tetramer-positive and negative populations were sorted with a MoFlo cell sorter (Cytomation Inc., Fort Collins, Colorado, USA).

Intracellular cytokine analysis using flow cytometry. Peptide-induced CTL lines were incubated in fresh FCS medium with peptide $(10 \mu \mathrm{M})$ and brefeldin A (10 $\mu \mathrm{g} / \mathrm{ml}$ ) (Sigma Chemical Co.) at $37^{\circ} \mathrm{C}$ for 4 hours. Cells were harvested and treated first with FACS lysing solution and then with FACS permeabilizing solution (BD Biosciences). The processed cells were resuspended in $50 \mu \mathrm{l}$ of FACS buffer and stained with PE-labeled antiCD8 (Caltag Laboratories Inc.), TC-labeled anti-CD4 (Caltag Laboratories Inc.), and FITC-labeled Ab's against one of the following cytokines: IFN- $\gamma$, TNF- $\alpha$, or IL-2 (BD Biosciences). An aliquot of the cells was also stained with FITC-labeled anti-CD8 (Caltag Laboratories Inc.), TC-labeled anti-CD4 (Caltag Laboratories Inc.), and PE-labeled Ab's against one of the following cytokines: IL-4 or IL-10 (BD Biosciences). In some experiments, IFN- $\gamma$ production of tetramer-binding cells was directly assessed by a combination of tetramer staining and intracellular staining for IFN- $\gamma$, as described $(14,19)$, with some modifications. The cells were incubated in fresh FCS medium with peptide $(10 \mu \mathrm{M})$ and brefeldin $\mathrm{A}(10 \mu \mathrm{g} / \mathrm{ml})$ at $37^{\circ} \mathrm{C}$ for 4 hours. Cells were harvested and resuspended in $50 \mu \mathrm{l}$ of FACS buffer and stained with PE-labeled tetramer for 30 minutes at room temperature in the dark, washed, and subsequently treated with FACS lysing solution and FACS permeabilizing solution. The processed cells were stained with FITC-labeled anti-IFN- $\gamma$ Ab's and TC-labeled anti-CD8 Ab's as described above. The stained cells were analyzed on a FACScan flow cytometer with CELLQUEST software (BD Biosciences).

Cytotoxicity assay. The HLA-A*0201+ lymphoblastoid $\mathrm{T} 2$ cell line was pulsed with appropriate peptides (10 $\mu \mathrm{M})$ at $37^{\circ} \mathrm{C}$ overnight and used as target cells. To assess the antigen-specific cytolytic activity of the CTL lines, a fluorescence-based cytotoxicity assay was carried out with DELFIA EuTDA cytotoxicity assay reagents (Wallac Oy, Turku, Finland), as described (6). In brief, the peptide-pulsed target cells were washed, labeled with TDA $\left(2,2^{\prime}: 6^{\prime}, 2^{\prime \prime}\right.$-terpyridine-6,6'-dicarboxylic acid), washed again, and resuspended in FCS medium. Five thousand target cells in a volume of $0.1 \mathrm{ml}$ were plated into each well of a 96-well round-bottom plate, followed by the addition of varying numbers of effector cells in $0.1 \mathrm{ml}$ of medium. After a 4-hour incubation at $37^{\circ} \mathrm{C}$, the plates were centrifuged and a $20-\mu$ l portion of the supernatant from each well was transferred to a 96-well microtiter plate containing $200 \mu$ l of DELFIA europium
(Eu) solution in each well. The Eu forms a stable complex with released TDA in the mixture and generates fluorescence. The fluorescence of EuTDA was measured by a time-resolved fluorometer (1420 VECTOR; Wallac Oy). Percentage of cytotoxicity was calculated by the formula: $100 \times$ (release in assay - spontaneous release)/(maximum release - spontaneous release). Maximum release was determined by the lysis of target cells in triplicate wells with DELFIA lysis buffer (Wallac Oy). Spontaneous release was measured by incubating target cells in triplicate wells in the absence of effector cells. In all experiments, spontaneous release was less than $15 \%$ of maximum release. Results are reported herein as the mean of triplicate values.

Estimation of specific $T$ cell frequency based on the number of cell division. The frequency of peptide-specific $\mathrm{CD}^{+} \mathrm{T}$ cells was analyzed as described previously (20), with some modification. In brief, an aliquot of CFSE-stained cells were suspended into the FCS medium and cultured at $37^{\circ} \mathrm{C}$. On day 7 , the cells were stimulated with $5 \mu \mathrm{M}$ of phytohemagglutinin$\mathrm{P}$ (PHA-P) and $10 \mathrm{U} / \mathrm{ml}$ of rIL-2 and further incubated at $37^{\circ} \mathrm{C}$ for another 7 days. Polyclonal stimulation of CFSE-stained cells with PHA-P and IL- 2 results in cell division accompanied by $50 \%$ dilution of CFSE per cell division in the cells, allowing determination of the mean CFSE intensity of each generation. These values were used to calculate the average number of cell division(s) in cell cultures stimulated with peptides. Precursor frequency was estimated by dividing the number of amplified tetramer-positive cells by $2^{x}$, where $x$ is the average number of cell divisions, to determine the absolute number of tetramer-positive precursors and then dividing this value by the total number of cells analyzed.

Statistical analysis. Values were statistically analyzed using the unpaired $t$ test.

\section{Results}

Peptide-loaded HLA-A*0201 tetramers bind specifically to $H L A-A * 0201-$ restricted CTLs. The specificity of tetramers binding to Ag-specific TCRs was verified using specific CTL lines induced by in vitro stimulation of PBMCs with peptide antigens. Aliquots of PBMCs from an HLA-A*0201+ PBC patient were cocultured with APCs pulsed with either PDC-E2 ${ }_{159-167}$ peptide or Flu MP S8-66 peptide for 14 days to expand peptide-specific $\mathrm{CD}^{+} \mathrm{T}$ cells in vitro. The peptide-specific CTL activity of the respective CTL lines was confirmed by a cytotoxicity assay (data not shown). Aliquots of these in vitroexpanded CTL lines were stained with either PE-labeled PDC-E2 $2_{159-167}$ tetramer or PE-labeled Flu $\mathrm{MP}_{58-66}$ tetramer, along with FITC-labeled anti-CD8 Ab's and TC-labeled anti-CD4 Ab's and analyzed by flow cytometry (Figure 1). In the PDC-E2 peptide-primed CTL line, there was a distinct population $(0.66 \%)$ of $\mathrm{CD}^{+} \mathrm{T}$ cells that stained positively with PDC-E2 $159-167$ tetramer, while no $(<0.1 \%)$ Flu $\mathrm{MP}_{58-66}$ tetramer-binding population was detected (Figure 1, a and c). On the 

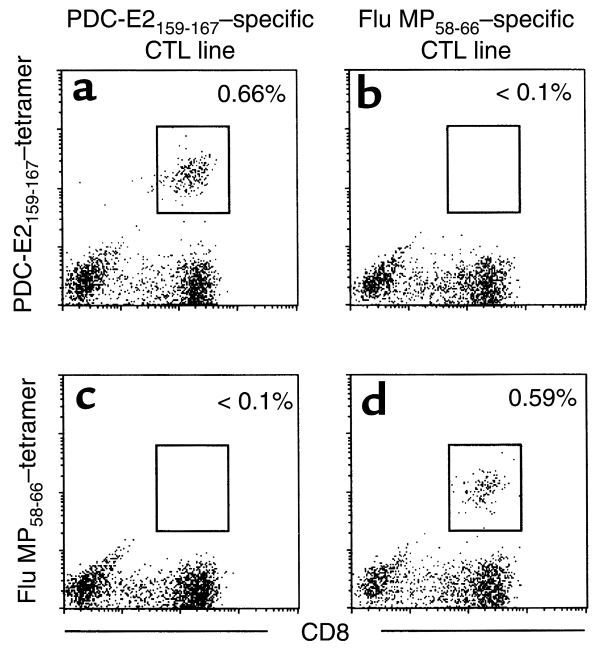

\section{Figure 1}

Verification of the binding specificity of peptide-MHC tetramers. PBMCs from an HLA-A* $0201^{+} \mathrm{PBC}$ patient were cocultured with PDC-

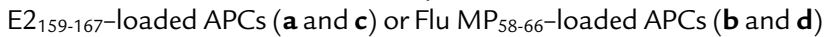
for 14 days. The cells were stained with the HLA-A*0201 tetramer loaded with PDC-E2 ${ }_{159-167}(\mathbf{a}$ and $\mathbf{b}$ ) or the HLA-A*0201 tetramer loaded with Flu MP58-66 (c and $\mathbf{d}$ ) in addition to anti-CD4 and antiCD8 Ab's, followed by flow cytometric analysis. Displayed in the dot plots are cells gated at lymphocyte population by forward scattering and side scattering and the CD4- population. The cells within the box are considered positive. The number next to the box is the percentage of positively stained cells in the lymphocyte population.

other hand, in the Flu MP peptide-primed CTL lines, there was a distinct population $(0.59 \%)$ of $\mathrm{CD}^{+} \mathrm{T}$ cells positively stained with Flu $\mathrm{MP}_{58-66}$ tetramer, while no $(<0.1 \%)$ binding was observed by the PDC-E $2_{159-167}$ tetramer (Figure 1, b and d). These experiments were repeated for both tetramers with independently generated CTL lines from six different HLA- $\mathrm{A}^{*} 0201^{+}$individuals, which verified the binding specificity of the tetramers. The frequencies of the PDC-E2 $2_{159-167}$ tetramer-positive $\mathrm{CD}^{+}$cells after in vitro stimulation of PBMCs from 12 PBC patients with PDC-E2 ${ }_{159-167}$ are summarized in Table 1. It is important to note that the frequency of PDC-E2 $2_{159-167}$ tetramer-positive $\mathrm{CD}^{+}$ cells were below the level of detection in the ex vivo-obtained $\mathrm{PBMCs}$ from all $12 \mathrm{PBC}$ patients. The frequency of PDC-E2 ${ }_{159-167}$ tetramer-positive $\mathrm{CD} 8^{+}$cells after in vitro stimulation with PDC-E2 $2_{159-167}$ was below the level of detection in PBMCs from all 12 control individuals, including four HLA-A* $0201^{+}$healthy controls, four HLA-A* $0201^{+}$patients with other chronic liver diseases, and four HLA-A2- patients with PBC (Table 1). The frequencies of Flu MP $\mathrm{MP}_{5866}$ tetramer-positive $\mathrm{CD}^{+}$cells after in vitro stimulation of PBMCs with Flu MP 58-66 $_{\text {peptide were between less than } 0.1 \%}$ and $10 \%$, presumably reflecting a history of infection or immunization in each patient with PBC. The frequency of Flu $\mathrm{MP}_{58-66}$ tetramer-positive $\mathrm{CD}^{+}$cells in the ex vivo-obtained PBMCs were between less than $0.1 \%$ and $0.2 \%$, similar to the healthy donors in a previous report (21).
Effector functions of PDC-E2 159-167.peptide-induced CTL lines. To more fully characterize the autoreactive CTL lines specific for PDC-E2, a CTL line was prepared by stimulating PBMCs from an HLA-A*0201 ${ }^{+} \mathrm{PBC}$ patient with PDC-E2 ${ }_{159-167}$ peptide-pulsed APCs in vitro. The cells were split into aliquots and assessed for cytotoxicity, cytokine production, and tetramer binding, respectively. Target cells loaded with PDC-E2 ${ }_{159-167}$, but not with a control peptide, were lysed by the CTL lines at an effector/target (E/T) ratio as low as 10:1 (Figure 2a). When the cells were restimulated with PDC-E2 159-167 peptide in the presence of brefeldin A, followed by intracellular staining for IFN- $\gamma$, a distinct population $(0.42 \%)$ of IFN- $\gamma$-producing cells was detected within the $\mathrm{CD}^{+} \mathrm{T}$ cell population (Figure $2 \mathrm{~b}$ ). The peptide-induced $\mathrm{T}$ cell lines were also stained with the PDC-E2 $2_{159-167}$ tetramer along with $\mathrm{mAb}$ to CD8 and CD4 and analyzed by flow cytometry. A distinct tetramer-positive $\mathrm{CD}^{+}$population $(0.82 \%)$ was detected in PDC-E2 peptide-specific CTL lines (Figure 2c). The frequencies of the tetramer-positive $\mathrm{CD}^{+}$cells, IFN- $\gamma$-producing $\mathrm{CD}^{+}$cells, and percentage of cytotoxicity of the CTL lines from eight HLA-A*0201+ patients with PBC are summarized in Tables 1 and 2. The frequency of the tetramer-positive cells was always higher than that of the IFN- $\gamma$-producing cells in each of the CTL lines studied.

Specific cytotoxicity of the PDC-E2 $2_{159-167}$ tetramer-reactive cells. In efforts to confirm that the tetramer-reactive cells represent true MHC class I-restricted autoreactive cytotoxic $\mathrm{T}$ cells, highly enriched populations of both PDC-E $2_{159-167}$ tetramer-positive and -negative $\mathrm{CD}^{+} \mathrm{T}$ cells were obtained by flow-cytometric sorting from PDC-E2 $2_{159-167}$ peptide-induced CTL lines. The sorted cells were used as effector cells in a cytotoxicity assay.

\section{Table 1}

Tetramer staining of PDC-E2 specific CD8 T cells after in vitro stimulation of PBMCs with PDC-E2 ${ }_{159-167}$

$\begin{array}{lccc}\text { Sample } & \begin{array}{c}\text { PDC-E2 } \text { 159-167 tetramer }^{+} \\ \text {CD8 }{ }^{+} \text {cells (\%) }\end{array} & \text { Sample } & \begin{array}{c}\text { PDC-E2 } \\ \text { CD9-167tetramer } \\ \text { CD8 }{ }^{+} \text {cells }(\%)\end{array} \\ \text { PBC 2 } & 0.52 & \text { CLD } 1^{A} 1 & <0.1 \\ \text { PBC 3 } & 1.13 & \text { CLD 2 } & <0.1 \\ \text { PBC 5 } & 0.53 & \text { CLD 3 } & <0.1 \\ \text { PBC 8 } & 0.13 & \text { CLD 4 } & <0.1 \\ \text { PBC 9 } & 0.14 & \text { Normal 1 } & <0.1 \\ \text { PBC 10 } & 0.24 & \text { Normal 2 } & <0.1 \\ \text { PBC 13 } & 0.82 & \text { Normal 3 } & <0.1 \\ \text { PBC 14 } & 0.36 & \text { Normal 4 } & <0.1 \\ \text { PBC 15 } & 0.66 & \text { A2 neg PBC 25 } & <0.1 \\ \text { PBC 16 } & 0.27 & \text { A2 neg PBC 26 } & <0.1 \\ \text { PBC 17 } & 0.12 & \text { A2 neg PBC 27 } & <0.1 \\ \text { PBC 18 } & 1.20 & \text { A2 neg PBC 28 } & <0.1\end{array}$

Mean \pm SD $\quad 0.42 \pm 0.33^{C}$

${ }^{A} C L D=$ chronic liver disease controls ${ }^{B} A 2$ neg $P B C=H L A$ A2 negative $P B C$ CValues for PBC patients were significantly $(P<0.0001)$ higher than those from controls (CLD, Normals, and A2 neg PBC). 
a

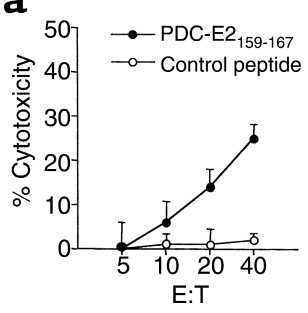

b

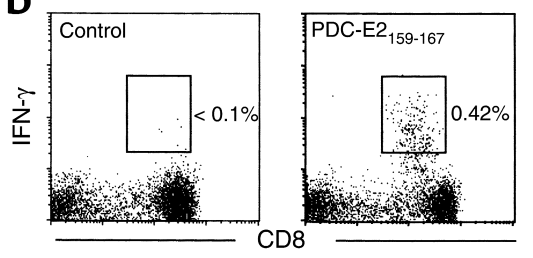

C

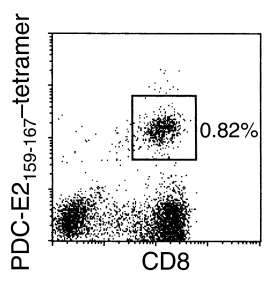

\section{Figure 2}

Identification and functional analysis of PDC-E2 ${ }_{159-167-s p e c i f i c ~ C D 8}{ }^{+}$T cells in the peptide-induced CTL line. PBMCs from an HLA-A*0201+ PBC patient were cocultured with PDC-E2 ${ }_{159-167}$-loaded APCs for 14 days to generate a CTL line. (a) The CTL line was tested for cytotoxicity against PDC-E2 ${ }_{159-167}$ peptide- or control peptide-loaded T2 targets at different E/T ratios. HBc 18-27, an HLA-A*0201-restricted irrelevant epitope, was used as control. Displayed are the mean specific lysis of triplicate testing. (b) The CTL line was restimulated with PDC-E2 ${ }_{159-167}$ peptide or control peptide in the presence of brefeldin A, followed by intracellular staining for IFN- $\gamma$. Displayed in the dot plots are cells gated at lymphocyte population by forward scattering and side scattering and the CD4- population. The number next to the box is the percentage of IFN- $\gamma$-producing cells in the lymphocyte population. (c) The CTL line was stained with PDC-E2 ${ }_{159-167}$ tetramer and Ab's against CD4 and CD8, followed by flow cytometric analysis. Displayed in the dot plots are cells gated at lymphocyte population by forward scattering and side scattering and the CD4- population. The number next to the box is the percentage of positively stained cells in the lymphocyte population.

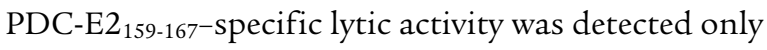
in the tetramer-positive population, not in the tetramer-negative population. Representative data from a PBC patient are shown in Figure 3. A highly enriched population of Flu $\mathrm{MP}_{58-66}$ tetramer-positive $\mathrm{CD}^{+} \mathrm{T}$ cells, prepared in the same way from Flu $\mathrm{MP}_{58-66}$ peptide-induced CTL lines, showed greater than $80 \%$ of Flu $\mathrm{MP}_{58-66^{-}}$specific lytic activity at an $\mathrm{E} / \mathrm{T}$ ratio of 10 (data not shown).

Cytokine production of the PDC-E2 $159-167$ tetramer-reactive cells. The frequency of PDC-E2 ${ }_{159-167}$ tetramer-positive cells was constantly higher than IFN- $\gamma$-producing cells in response to restimulation with PDC-E2 $2_{159-167}$ peptide; this suggests the presence of PDC-E $2_{159-167}$ tetramer-reactive cells that do not respond with IFN- $\gamma$ production after short-term restimulation with the PDC-E2 ${ }_{159-167}$ peptide. Thus, the ability of individual tetramer-binding cells to produce IFN- $\gamma$ in response to the PDC-E2 ${ }_{159-167}$ peptide stimulation was assessed. PDC-E2 ${ }_{159-167-}$ specific CTL lines from HLA-A*0201+ PBC patients were restimulated with PDC-E2 ${ }_{159-167}$ or a control peptide in the presence of brefeldin A, stained with the PDC-E2 $2_{159-167}$ tetramer, fixed and permeabilized, and stained for intracellular IFN- $\gamma$ along with $\mathrm{mAb}$ against CD8. For purposes of control, aliquots of the same PBMCs were cocultured $w$ ith APCs pulsed with Flu MP 58-66 peptide for 14 days to expand Flu $\mathrm{MP}_{58-66}$ peptide-specific $\mathrm{CD}^{+} \mathrm{T}$ cells in vitro. When the Flu MP $\mathrm{M}_{58-66}$-specific CTL lines were restimulated with Flu $\mathrm{MP}_{58-66}$ peptide, virtually all (> 99\%) the Flu $\mathrm{MP}_{58-66}$ tetramer-positive cells produced high levels of IFN- $\gamma$ (Figure $4 b$ ). On the other hand, when the PDC-E2 159-167-specific CTL lines were stimulated with PDC-E2 $2_{159-167}$ peptide, two distinct tetramer-positive populations with different intensity of tetramer staining were identified. One of them was responsive to the specific peptide stimulation, as indicated by the downmodulated tetramer staining (14, 19 ) as well as production of IFN- $\gamma$. However, the level of IFN- $\gamma$ production by these cells was lower than that of the Flu MP 58-66 $_{\text {tetramer-positive cells. The other }}$ tetramer-positive population was nonresponsive to peptide stimulation, as indicated by the unchanged tetramer-staining intensity and the lack of IFN- $\gamma$ production (Figure 4a). Similar results were reproduced in six patients with PBC (data not shown). These results suggest that the capability of IFN- $\gamma$ production was lower and heterogeneous within the PDC-E2 $2_{159-167}$ tetramer-positive population. It is also important to note that the mean density of tetramer-positive cells for the Flu MP peptide was lower than the PDC-E2

\section{Table 2}

IFN- $\gamma$ production and cytotoxicity of PDC-E2 specific CD8 T cells after in vitro stimulation of PBMCs with PDC-E2 $159-167$

\begin{tabular}{lcc} 
Sample & $\begin{array}{c}\text { IFN- } \gamma \text { producing } \\
\text { CD8 }{ }^{+} \text {cells }(\%)\end{array}$ & $\begin{array}{c}\text { Specific cytotoxicity (\%) } \\
(\mathrm{E}: \mathrm{T}=40)\end{array}$ \\
PBC 2 & 0.30 & 20 \\
PBC 3 & 0.73 & 43 \\
PBC 5 & 0.28 & 28 \\
PBC 10 & 0.15 & 17 \\
PBC 13 & 0.42 & 25 \\
PBC 14 & 0.13 & 10 \\
PBC 16 & 0.10 & 12 \\
PBC 18 & 0.83 & 48 \\
Mean \pm SD & $0.37 \pm 0.27^{A}$ & $25.38 \pm 13.8^{B}$ \\
CLD 1 & & $<5$ \\
CLD 2 & $<0.1$ & $<5$ \\
Normal 1 & $<0.1$ & $<5$ \\
Normal 2 & $<0.1$ & $<5$ \\
A2 neg PBC 25 & $<0.1$ & $<5$ \\
A2 neg PBC 26 & $<0.1$ & $<5$ \\
\hline
\end{tabular}

AFrequencies of IFN- $\gamma$ producing $C D 8 \mathrm{~T}$ cells in $\mathrm{PBC}$ patients were significantly $(P<0.0001)$ higher than those from controls (CLD, Normals, and A2 neg PBC). BValues of specific cytotoxicity in PBC patients were significantly $(P<0.0001)$ higher than those from controls (CLD, Normals, and $A 2$ neg $P B C$ ). ${ }^{C} C L D=$ chronic liver disease controls ${ }^{\mathrm{D}} \mathrm{A} 2$ neg $\mathrm{PBC}=\mathrm{HLA} A 2$ negative $\mathrm{PBC}$. 
a

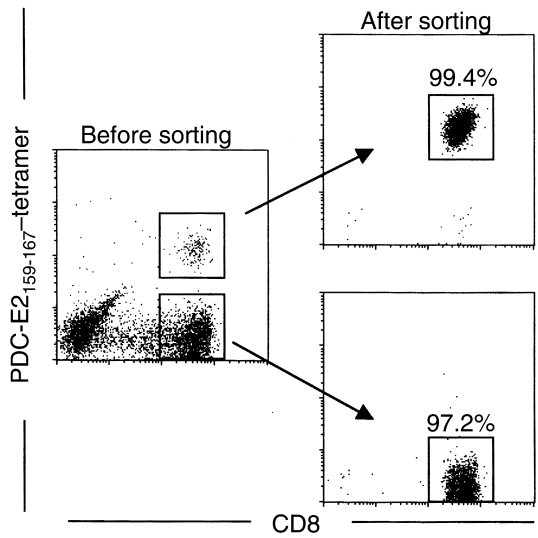

b

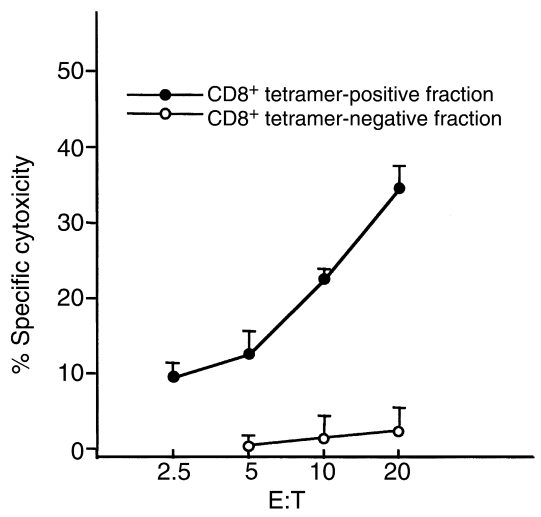

\section{Figure 3}

The specific cytotoxicity of PDC-E2 $159-167$ peptide-induced cell line is mediated by tetramer-positive CD8 ${ }^{+}$T cells. PBMCs from an HLA$A^{*} 0201^{+}$PBC patient were cocultured with PDC-E2 ${ }_{159-167}$-loaded APCs for 14 days to generate a CTL line. (a) Cells were stained with the PDC-E2 159-167 tetramer and Ab's against CD4 and CD8, followed by flow cytometric cell sorting to isolate tetramer-positive CD8 ${ }^{+}$and tetramer-negative $C D 8^{+}$populations. (b) The sorted cells were tested for their cytotoxicity against T2 target cells loaded with PDC-E2 $159-167$ peptide or a control peptide $\mathrm{HBc}_{18-27}$ at different $\mathrm{E} / \mathrm{T}$ ratios. Displayed is the mean specific lysis of triplicate testing.

peptide-specific $\mathrm{CD}^{+} \mathrm{T}$ cells, denoting differences in the relative downregulation of TCRs in the two antigen-specific CTL lines. These data also suggest that part of the PDC-E2 ${ }_{159-167}$ tetramer-positive population is anergized. The ability of the PDC-E2 $2_{159-167}$ peptidespecific CTLs to produce other cytokines in response to the PDC-E2 $159-167$ peptide stimulation was also assessed. However, no detectable level of cells producing TNF- $\alpha$, IL-2, IL-4, or IL-10 was observed in any of four patients studied (data not shown).

$P D C-E 2_{159-167}$ tetramer-reactive cells in the liver. To assess whether PDC-E2 ${ }_{159-167-s p e c i f i c ~} \mathrm{CD} 8^{+} \mathrm{T}$ cells exist in end-stage PBC livers, LILs were isolated from explanted liver tissue of six HLA- $\mathrm{A}^{*} 0201^{+}$patients (PBC 19 to 24) undergoing liver transplantation. There were undetectable levels of PDC-E2 $2_{159-167}$ tetramer-positive $\mathrm{CD}^{+} \mathrm{T}$ cells in uncultured LIL samples. However, when the LILs from the explanted livers of the PBC patients were stimulated with APCs pulsed with PDCE2 ${ }_{159-167}$ peptide for 14 days to expand peptide-specific $\mathrm{CD}^{+} \mathrm{T}$ cells in vitro, tetramer-binding $\mathrm{CD}^{+} \mathrm{T}$ cells were readily detectable in each of the six PBC patients (Table 3). For comparison, LILs were isolated from the excess liver tissues donated by two healthy HLA$\mathrm{A}^{*} 0201^{+}$individuals (normal 5 to 6 ) intended for transplantation. These cells were also stimulated with APCs pulsed with PDC-E2 $2_{159-167}$ peptide for 14 days, followed by tetramer staining. No tetramer-positive $\mathrm{CD}^{+} \mathrm{T}$ cells were detected in the in vitro-cultured LILs from either donor (Table 3 ).

The frequency of PDC-E $2_{159-167}$ tetramer-reactive cells in the blood and liver. To estimate the frequency of PDC$\mathrm{E} 2_{159-167-s p e c i f i c ~} \mathrm{CD} 8^{+} \mathrm{T}$ cells in the peripheral blood and liver, PBMCs from 12 HLA-A*0201+ patients with PBC at different stages (PBC 1 to 12) and LILs from end-stage PBC liver of six HLA-A*0201+ PBC patients (PBC 19 to 24) were first stained with CFSE, then cocultured with APCs pulsed with PDC-E2 ${ }_{159-167}$ peptide for 14 days, to expand peptide-specific CD8 ${ }^{+}$ $\mathrm{T}$ cells. The induced CTL lines were stained with PDC-E2 ${ }_{159-167}$ tetramer, along with $\mathrm{mAb}$ to CD8, and analyzed by flow cytometry. A distinct population of $\mathrm{CD}^{+} \mathrm{T}$ cells positively stained with the PDC-E $2_{159-167}$ tetramer was detected in 10 of 12 of PBMCs and each of the six cases of LILs studied. Representative results of a PBMC sample and a LIL sample are shown in Figure 5, $a$ and $b$. The number of cell divisions undergone by the tetramer-positive cells was quantitated relative to the intensity of CFSE fluorescence and was similar in PBMCs and LILs, with values of $6.20 \pm 0.79$ compared with $6.50 \pm 0.55$, respectively. The frequencies of the PDC-E2 $159-167$ tetramer-positive cells in the uncultured PBMCs were calculated as

\section{Table 3}

Tetramer staining of PDC-E2 specific CD8 T cells after in vitro stimulation of LILs with PDC-E2 $159-167$
Sample

PBC 19

PBC 20

$\mathrm{PBC} 21$

$\mathrm{PBC} 22$

$\mathrm{PBC} 23$

PBC 24

Mean \pm SD

Normal 5

Normal 6
AValues for PBC patients were significantly $(P=0.0047)$ higher than those from controls 
a

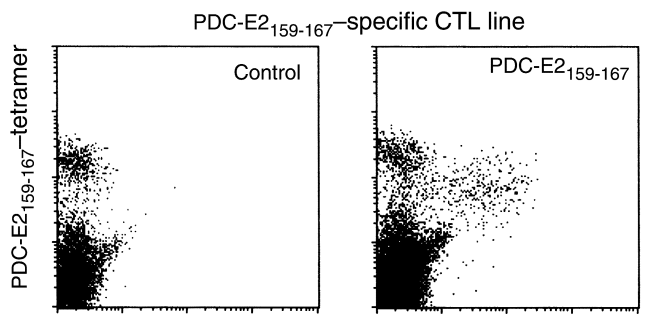

b

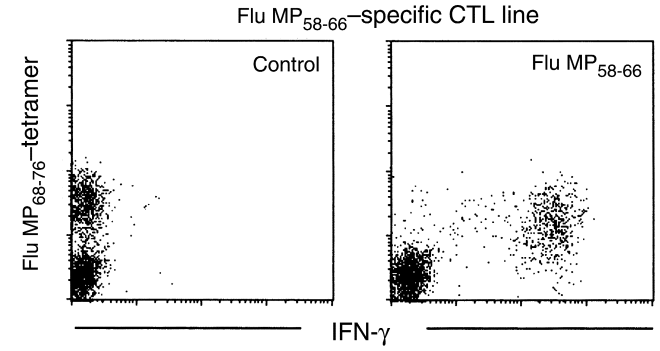

described above. More than $90 \%$ of the tetramer-positive cells was identified within the single peak of the cell division in all cases studied. The results for 12 PBMC samples and six LIL samples from HLA$\mathrm{A} 2 * 0201^{+} \mathrm{PBC}$ patients, as well as eight PBMC samples and two LIL samples from HLA- A2 ${ }^{*} 0201^{+}$control, are summarized in Table 4 . The average frequency of PDC-E2 159 -167 tetramer-positive CD8 T cells in peripheral blood from $12 \mathrm{PBC}$ patients was $3.58 \pm 3.26 \times 10^{-5}$, while that in the six end-stage PBC liver was $4.14 \pm 0.95 \times 10^{-4}$, over tenfold and significantly higher than that in the peripheral blood $(P<0.0001)$ (Table 4). These data suggest that PDC-

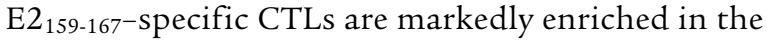
liver. In addition, it is important to note that the frequency of PDC-E $2_{159-167}$-specific CD8 ${ }^{+} \mathrm{T}$ cells from peripheral blood were significantly higher in earlier
Figure 4

Antigen specificity of IFN- $\gamma$ production by $C D 8^{+} T$ cells for PDC-E2 autoantigen or influenza viral antigen. (a) PDC-E2 ${ }_{159-167}$ peptide-induced CTL lines derived from a PBC patient was restimulated with PDC-E2 ${ }_{159-167}$ peptide or a control peptide in the presence of brefeldin $A$. The cells were first stained with the PDC-E2 159-167 tetramer, followed by intracellular staining for IFN- $\gamma$. Displayed in the dot plots are cells gated at lymphocyte population by forward scattering and side scattering and the CD8 ${ }^{+}$population. (b) Flu $\mathrm{MP}_{58-66}$ peptide-induced CTL lines derived from the same PBC patient was restimulated with Flu $\mathrm{MP}_{58-66}$ peptide or a control peptide in the presence of brefeldin A. The cells were first stained with the Flu MP $\mathrm{MP}_{586}$ tetramer, followed by intracellular staining for IFN- $\gamma$. Displayed in the dot plots are cells gated at lymphocyte population by forward scattering and side scattering and the $\mathrm{CD} 8^{+}$population.

stages I and II of PBC $\left(6.75 \pm 2.21 \times 10^{-5}\right)$ as compared with later stages III and IV $\left(1.31 \pm 1.33 \times 10^{-5}\right.$, $P<0.0003)$.

\section{Discussion}

CTLs play a pivotal role in host defense against viral infections and tumor development. In addition, several lines of evidence suggest that autoreactive CTLs are involved in the pathogenesis of human autoimmune diseases as well as animal models of autoimmune diseases (22-26). In a previous study we demonstrated that PDC-E $2_{159-167^{-}-\text {spe- }}$ cific CTL lines can be induced from PBMCs in HLA$A^{*} 0201^{+} \mathrm{PBC}$ patients (6). In the present study, we have further characterized the peptide-induced CTLs using a PDC-E2 ${ }_{159-167-H L A-A}$ 0201 tetramer reagent. Our results indicate that a distinct population of PDC-E2 ${ }_{159-167}$ tetramer-reactive cells can be detected after in vitro stimulation of PBMCs from HLA-A* $0201^{+}$PBC patients, but not from controls. These tetramer-reactive cells demonstrate specific cytotoxicity against PDC-E $2_{159-167}$-pulsed target cells, as shown by CTL assays using purified tetramer-positive and -negative cells. The in vitro-expanded PDC-E2-specific CD8 ${ }^{+}$T cells synthesized IFN- $\gamma$ but

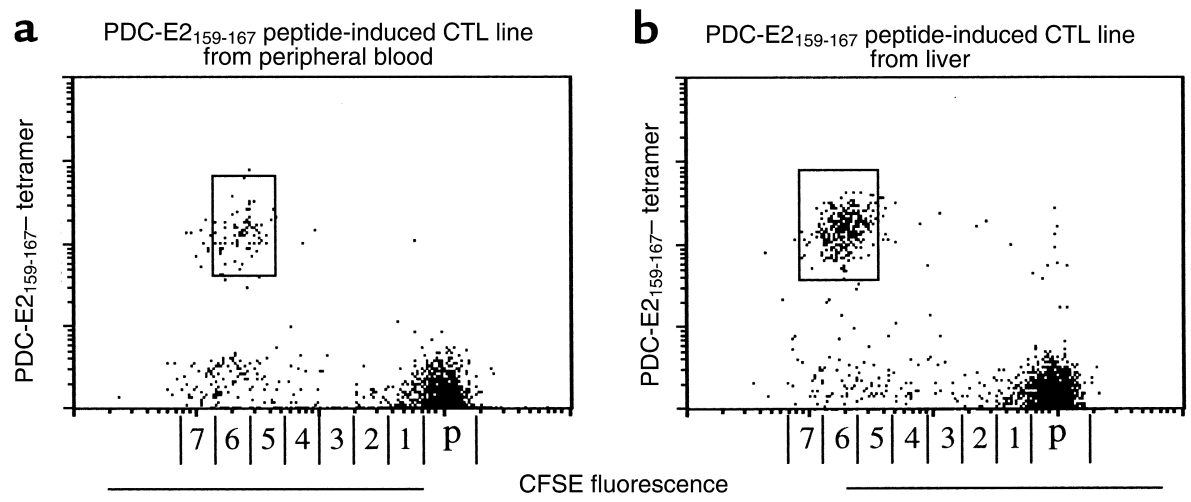

\section{Figure 5}

In vitro expansion of PDC-E2 ${ }_{159-167}$ tetramer-reactive cells. PBMCs $(\mathbf{a})$ and LILs $(\mathbf{b})$ from HLA-A*0201+ PBC patients were stained with CFSE and cocultured with PDC-E2 $159-167$-loaded APCs for 14 days. The cells were stained with the PDC-E2 tetramer and anti-CD8 Ab's, followed by flow cytometric analysis. Displayed in the dot plots are cells gated at lymphocyte population by forward scattering and side scattering and the $\mathrm{CD} 8^{+}$population. The number next to the box is the percentage of tetramer-stained cells in the lymphocyte population. The horizontal axis is labeled with numbers corresponding to cell divisions and with " $p$ " depicting the undivided parent cell population. This scale was calculated from the distinct CFSE fluorescence peaks produced by polyclonal stimulation with PHA-P and IL-2. 
Table 4

Precursor frequency of PDC-E2-specific CD8 T cells

\begin{tabular}{|c|c|c|c|c|c|}
\hline Sample & Stage & Source & $\begin{array}{c}\text { Frequency } \\
(\text { per } 100,000)\end{array}$ & $\begin{array}{l}\text { Average frequency } \\
\quad(\text { Mean } \pm \text { SD) }\end{array}$ & $\begin{array}{l}\text { Average frequency } \\
\quad(\text { Mean } \pm \text { SD })\end{array}$ \\
\hline PBC 1 & 1 & blood & 4.68 & & \\
\hline PBC 2 & 1 & blood & 7.92 & & \\
\hline PBC 3 & 1 & blood & 8.83 & ${ }^{A} 6.75 \pm 2.21 \times 10^{-5}$ & \\
\hline PBC 4 & II & blood & 4.06 & & \\
\hline PBC 5 & $\|$ & blood & 8.28 & & \\
\hline PBC 6 & III & blood & 1.37 & & B $3.58 \pm 3.26 \times 10^{-5}$ \\
\hline PBC 7 & III & blood & undetectable & & \\
\hline PBC 8 & III & blood & 2.10 & & \\
\hline PBC 9 & IV & blood & 0.54 & ${ }^{\mathrm{A}} 1.31 \pm 1.33 \times 10^{-5}$ & \\
\hline PBC 10 & IV & blood & 3.75 & & \\
\hline PBC 11 & IV & blood & 1.40 & & \\
\hline PBC 12 & IV & blood & undetectable & & \\
\hline CLD 1 & & blood & undetectable & & \\
\hline CLD 2 & & blood & undetectable & & \\
\hline CLD 3 & & blood & undetectable & & \\
\hline CLD 4 & & blood & undetectable & & \\
\hline Normal 1 & & blood & undetectable & & \\
\hline Normal 2 & & blood & undetectable & & \\
\hline Normal 3 & & blood & undetectable & & \\
\hline Normal 4 & & blood & undetectable & & \\
\hline PBC 19 & IV & liver & 42.70 & & \\
\hline PBC 20 & IV & liver & 38.50 & & \\
\hline PBC 21 & IV & liver & 52.50 & B $4.14 \pm 0.95 \times 10^{-4}$ & \\
\hline PBC 22 & IV & liver & 47.15 & & \\
\hline PBC 23 & IV & liver & 24.53 & & \\
\hline PBC 24 & IV & liver & 42.81 & & \\
\hline Normal 5 & & liver & undetectable & & \\
\hline Normal 6 & & liver & undetectable & & \\
\hline
\end{tabular}

AP $<0.0003$ comparing stage I and II to stage III and IV. ${ }^{B} P<0.0001$ comparing blood to liver in patients with PBC.

not TNF- $\alpha$ and IL-2, the other two major type 1 cytokines produced by $\mathrm{CD} 4^{+} \mathrm{Th} 1$ cells and $\mathrm{CD} 8^{+} \mathrm{CTL}$ specific for viral antigens. In addition, the synthesis of IL-4 and IL-10, major type 2 cytokines, were not observed in these cells.

In the present study, we demonstrate that the ability of the PDC-E2-specific CTLs detected by PDC-E2 159-167 tetramer to produce IFN- $\gamma$ is lower and heterogeneous as compared with influenza-specific CTLs. One possible explanation is that some of the peptide-specific $\mathrm{CD}^{+} \mathrm{T}$ cells may lose their capability to synthesize cytokines during in vitro culturing. However, our finding that virtually all of the Flu $\mathrm{MP}_{58-66}$-specific $\mathrm{CD}^{+} \mathrm{T}$ cells from the same patients were capable of producing IFN- $\gamma$ argues against this explanation, suggesting that heterogeneity in cytokine profile may be an intrinsic character of the autoantigen-specific $\mathrm{CD} 8^{+} \mathrm{T}$ cells that is different from virus-specific $\mathrm{CD} 8^{+} \mathrm{T}$ cells. In contrast to virus-specific immunity, the immune response to tumor cells is often less vigorous and results in CTLs characterized by lower avidity and decreased functional capacity, especially when the tumor Ags are normal self proteins (27-29). Tumor-specific CTLs and autoreactive CTLs may share common features because they both recognize self-antigen. In fact, it is plausible that the epitope derived from endogenous PDC-E2 could be expressed in association with the MHC molecule on all cells, even at a low level, which may lead autoreactive $T$ cells to anergy. There is a relatively small increase in cytotoxicity by

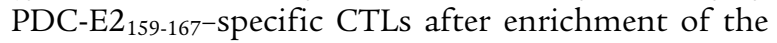
tetramer-positive cells, probably in part because of the modulation of the effector function of the cells by PDCE2 159-167 tetramer directly bound on cells. The low lytic activity of highly enriched $\mathrm{CD}^{+}$cell line specific for PDC-E2 159-167, compared with highly enriched Flu

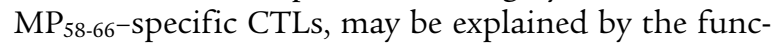

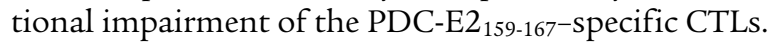

An alternate interpretation of the functional impairment, in terms of cytokine production, is that the autoantigen-derived peptide-specific PDC-E $2_{159-167}$ is not identical to the epitope that induces the primary $\mathrm{CD}^{+} \mathrm{T}$ cell response, but a molecular mimic of the original immunogen. The primary $\mathrm{CD}^{+}$response may be elicited by an epitope of an unknown xenobiotic agent, such as a chemical or a virus (30). In this case, the 
PDC-E $2_{159-167}$ epitope may actually be recognized by the xenobiotic-specific $\mathrm{CD}^{+} \mathrm{T}$ cells as an altered peptide ligand, which is known to be able to induce differential effector functions on the $\mathrm{CD}^{+} \mathrm{T}$ cells (31). This interpretation is consistent with the view that molecular mimicry may be operating in PBC.

Characterization of the intrahepatic $\mathrm{T}$ cell response is important for our understanding of the mechanisms underlying liver pathology. In the present study, PDCE2 ${ }_{159-167^{-}}$-specific CTL lines have also been successfully induced from LILs of HLA-A* $0201^{+}$patients with endstage PBC. By combination of CFSE staining and tetramer staining, we estimate that the frequency of

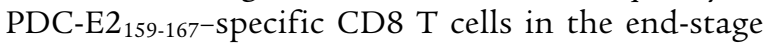
PBC liver is $4.14 \times 10^{-4}$, while that in the periphery of $\mathrm{PBC}$ patients is $3.58 \times 10^{-5}$. These data suggest that PDC-E $2_{159-167-s p e c i f i c ~} \mathrm{CD}^{+} \mathrm{T}$ cells are enriched approximately tenfold in the liver. There is also the possibility that part of the PDC-E2 ${ }_{159-167-}$-specific CTLs may stop dividing during in vitro culture due to anergy. However, most of the tetramer-positive cells were identified within a single peak of cell division, suggesting that PDC-E $2_{159-167}$-specific CTLs may undergo similar division. In an earlier study using limiting dilution analysis, the frequency of peripheral $\mathrm{CD}^{+}$helper $\mathrm{T}$ cells specific for the PDC-E2 $2_{163-176}$ epitope in PBC patients was estimated to be $3.66 \times 10^{-7}$, while that in the liver was $1.66 \times 10^{-5}$ to $4.13 \times 10^{-5}, 100$-fold enrichment in the liver as compared with PBMC's (13). Taken together, these studies indicate that there is selective enrichment for both PDC-E2-specific $\mathrm{CD}^{+}$and $\mathrm{CD} 8^{+}$ autoreactive $T$ cells localized to the site of tissue injury in patients with PBC. The discrepancy of the level of frequency between autoreactive $\mathrm{CD}^{+}$and $\mathrm{CD} 8^{+} \mathrm{T}$ cells could be due to the difference of the methods of detection $(32,33)$. Interestingly, the extent of enrichment in the PDC-E2-specific autoreactive $\mathrm{CD} 4^{+} \mathrm{T}$ cells is higher than $\mathrm{CD}^{+} \mathrm{T}$ cells. This difference cannot be readily explained by the differences in the techniques used in the previous and present study. On the other hand, if indeed the values are, in fact, true differences, this could reflect a more important role for $\mathrm{CD} 4^{+} \mathrm{T}$ cells in the pathogenesis of $\mathrm{PBC}$ in late disease. Further study of $\mathrm{CD}^{+} \mathrm{T}$ cells using class II tetramer is warranted and may provide additional information about the nature of the autoreactive $\mathrm{CD} 4^{+} \mathrm{T}$ cells.

The estimated frequency of PDC-E2-specific $\mathrm{CD}^{+} \mathrm{T}$ cells from peripheral blood was significantly higher in early stages of PBC than in the more advanced disease stages. This is in agreement with a previous report showing that the frequency of peripheral blood $\mathrm{CD} 4^{+}$ $\mathrm{T}$ cells that respond to the helper $\mathrm{T}$ cell epitope 163-176 was significant higher in the early stage of $\mathrm{PBC}$ as compared with the advanced stage (13). In previous studies directly comparing the frequency of hepatitis $\mathrm{C}$ virus-specific (HCV-specific) $\mathrm{CD}^{+} \mathrm{T}$ cells in peripheral blood and liver, higher frequencies of HCVspecific $\mathrm{CD}^{+} \mathrm{T}$ cells were found in liver biopsies than in the blood of patients with chronic HCV infection
$(14,34)$. In another study using end-stage cirrhotic liver, the HCV-specific $T$ cell could not be found in the liver (35), suggesting that the level of virus-specific intrahepatic CTLs declines as the viral hepatitis progress. Supposing that declination of the diseasespecific CTL with the disease progression occurs in $\mathrm{PBC}$, the PDC-E2-specific CD8 ${ }^{+} \mathrm{T}$ cells in the liver may be even more abundant during the earlier stages of PBC and be playing an important role in disease development. In addition, the autoreactive CTL response in PBC may also involve other epitopes restricted by HLA$A^{*} 0201$, as well as epitopes restricted by other HLA molecules. Identification and characterization of these epitopes is required to generate a more complete picture of the overall CTL response in PBC during different disease stages.

In conclusion, this study provides evidence that PDC$\mathrm{E} 2-$ specific $\mathrm{CD}^{+} \mathrm{T}$ cells are enriched in the liver. In addition to our data on $\mathrm{CD}^{+} \mathrm{T}$ cells, previous studies have shown that high numbers of PDC-E2-specific $\mathrm{CD}^{+}$ helper T cells, as well as B cells that produce PDC-E2 autoantibodies, are enriched in the PBC liver $(13,36)$. We believe that anti-PDC-E2 Ab's may form immune complexes with antigens and be taken up by APCs, including DCs (6). In addition, autoreactive B cells may be efficient APCs for priming $T$ cells by specific uptake of the autoantigen via surface Ig receptor as reported in other autoimmune diseases $(37,38)$. These cells may lead to the activation of autoreactive $\mathrm{CD} 4^{+}$and $\mathrm{CD}^{+} \mathrm{T}$ cells, which in turn mediate damage on bilary epithelial cells (BECs) as well as activating autoreactive $B$ cells to produce autoantibodies. Therefore the autoreactive $\mathrm{B}$ cells, $\mathrm{CD}^{+}$ $\mathrm{T}$ cells, and $\mathrm{CD}^{+} \mathrm{T}$ cells may collectively form an orchestrated autoimmune effector response that leads to the pathogenesis of PBC. More importantly, the response is focused on PDC-E2, the major mitochondrial antigen, lending further weight to the argument that either native modified PDC-E2 and/or a mimeotope resembling PDC$\mathrm{E} 2$ is instrumental in the etiology of disease.

\section{Acknowledgments}

This study was funded by NIH grant DK-39588.

1. Coppel, R.L., and Gershwin, M.E. 1995. Primary biliary cirrhosis: the molecule and the mimic. Immunol. Rev. 144:17-49.

2. Van de Water, J., et al. 1997. The role of T cells in primary biliary cirrhosis. Semin. Liver Dis. 17:105-113.

3. Colucci, G., Schaffner, F., and Paronetto, F. 1986. In situ characterization of the cell-surface antigens of the mononuclear cell infiltrate and bile duct epithelium in primary biliary cirrhosis. Clin. Immunol. Immunopathol. 41:35-42.

4. Van de Water, J., et al. 1995. Heterogeneity of autoreactive T cell clones specific for the $\mathrm{E} 2$ component of the pyruvate dehydrogenase complex in primary biliary cirrhosis. J. Exp. Med. 181:723-733.

5. Shimoda, S., Nakamura, M., Ishibashi, H., Hayashida, K., and Niho, Y. 1995. HLA DRB4 0101-restricted immunodominant T cell autoepitope of pyruvate dehydrogenase complex in primary biliary cirrhosis: evidence of molecular mimicry in human autoimmune diseases. J. Exp. Med. 181:1835-1845.

6. Kita, H., et al. 2002. Identification of HLA-A2-restricted CD8(+) cytotoxic $\mathrm{T}$ cell responses in primary biliary cirrhosis: $\mathrm{T}$ cell activation is augmented by immune complexes cross-presented by dendritic cells. J. Exp. Med. 195:113-123.

7. Huseby, E.S., et al. 2001. A pathogenic role for myelin-specific CD8(+) T cells in a model for multiple sclerosis. J. Exp. Med. 194:669-676. 
8. Sun, D., et al. 2001. Myelin antigen-specific CD8+ T cells are encephalitogenic and produce severe disease in C57BL/6 mice. J. Immunol. 166:7579-7587.

9. Wong, F.S., et al. 1999. Identification of an MHC class I-restricted autoantigen in type 1 diabetes by screening an organ-specific cDNA library. Nat. Med. 5:1026-1031.

10. Chou, Y.K., et al. 1992. Frequency of T cells specific for myelin basic protein and myelin proteolipid protein in blood and cerebrospinal fluid in multiple sclerosis. J. Neuroimmunol. 38:105-113.

11. Zhang, J., et al. 1994. Increased frequency of interleukin 2-responsive T cells specific for myelin basic protein and proteolipid protein in peripheral blood and cerebrospinal fluid of patients with multiple sclerosis. J. Exp. Med. 179:973-984.

12. Link, H., et al. 1992. Virus-reactive and autoreactive T cells are accumulated in cerebrospinal fluid in multiple sclerosis. J. Neuroimmunol. 38:63-73.

13. Shimoda, S., et al. 1998. Identification and precursor frequency analysis of a common $\mathrm{T}$ cell epitope motif in mitochondrial autoantigens in primary biliary cirrhosis. J. Clin. Invest. 102:1831-1840.

14. He, X.S., et al. 1999. Quantitative analysis of hepatitis C virus-specific CD8(+) $\mathrm{T}$ cells in peripheral blood and liver using peptide-MHC tetramers. Proc. Natl. Acad. Sci. USA. 96:5692-5697.

15. Nayersina, R., et al. 1993. HLA A2 restricted cytotoxic T lymphocyte responses to multiple hepatitis $B$ surface antigen epitopes during hepatitis B virus infection. J. Immunol. 150:4659-4671.

16. Garboczi, D.N., Hung, D.T., and Wiley, D.C. 1992. HLA-A2-peptide complexes: refolding and crystallization of molecules expressed in Escherichia coli and complexed with single antigenic peptides. Proc. Natl. Acad. Sci. USA. 89:3429-3433.

17. Altman, J.D., et al. 1996. Phenotypic analysis of antigen-specific T lymphocytes. Science. 274:94-96.

18. Sallusto, F., and Lanzavecchia, A. 1994. Efficient presentation of soluble antigen by cultured human dendritic cells is maintained by granulocyte/macrophage colony-stimulating factor plus interleukin 4 and downregulated by tumor necrosis factor alpha. J. Exp. Med. 179:1109-1118.

19. He, X.S., et al. 2001. Direct functional analysis of epitope-specific CD8+ $\mathrm{T}$ cells in peripheral blood. Viral. Immunol. 14:59-69.

20. Novak, E.J., Liu, A.W., Nepom, G.T., and Kwok, W.W. 1999. MHC class II tetramers identify peptide-specific human CD4(+) T cells proliferating in response to influenza A antigen. J. Clin. Invest. 104:R63-67.

21. Dunbar, P.R., et al. 1998. Direct isolation, phenotyping and cloning of low-frequency antigen-specific cytotoxic T lymphocytes from peripheral blood. Curr. Biol. 8:413-416.

22. Panina-Bordignon, P., et al. 1995. Cytotoxic T cells specific for glutamic acid decarboxylase in autoimmune diabetes. J. Exp. Med. 181:1923-1927.

23. Tsuchida, T., et al. 1994. Autoreactive CD8+ T-cell responses to human myelin protein-derived peptides. Proc. Natl. Acad. Sci. USA. 91:10859-10863

24. Pender, M.P., Csurhes, P.A., Houghten, R.A., McCombe, P.A., and Good, M.F. 1996. A study of human T-cell lines generated from multiple sclerosis patients and controls by stimulation with peptides of myelin basic protein. J. Neuroimmunol. 70:65-74.

25. D'Elios, M.M., et al. 2001. H(+),K(+)-atpase (proton pump) is the target autoantigen of Th1-type cytotoxic T cells in autoimmune gastritis. Gas troenterology. 120:377-386.

26. Fiorillo, M.T., Maragno, M., Butler, R., Dupuis, M.L., and Sorrentino, R 2000. CD8(+) T-cell autoreactivity to an HLA-B27-restricted self-epitope correlates with ankylosing spondylitis. J. Clin. Invest. 106:47-53.

27. Houghton, A.N. 1994. Cancer antigens: immune recognition of self and altered self. J. Exp. Med. 180:1-4.

28. Boon, T., et al. 1994. Genes coding for tumor-specific rejection antigens. Cold Spring Harb. Symp. Quant. Biol. 59:617-622.

29. Lee, P.P., et al. 1999. Characterization of circulating T cells specific for tumor-associated antigens in melanoma patients. Nat. Med. 5:677-685.

30. Long, S.A., et al. 2001. Immunoreactivity of organic mimeotopes of the E2 component of pyruvate dehydrogenase: connecting xenobiotics with primary biliary cirrhosis. J. Immunol. 167:2956-2963.

31. Evavold, B.D., and Allen, P.M. 1991. Separation of IL-4 production from Th cell proliferation by an altered $\mathrm{T}$ cell receptor ligand. Science. 252:1308-1310.

32. Murali-Krishna, K., et al. 1998. Counting antigen-specific CD8 T cells: a reevaluation of bystander activation during viral infection. Immunity. 8:177-187.

33. Callan, M.F., et al. 1998. Direct visualization of antigen-specific CD8+ T cells during the primary immune response to Epstein-Barr virus in vivo. J. Exp. Med. 187:1395-1402.

34. Grabowska, A.M., et al. 2001. Direct ex vivo comparison of the breadth and specificity of the $T$ cells in the liver and peripheral blood of patients with chronic HCV infection. Eur. J. Immunol. 31:2388-2394.

35. Valiante, N.M., et al. 2000. Life, activation and death of intrahepatic lymphocytes in chronic hepatitis C. Immunol. Rev. 174:77-89.

36. Bjorkland, A., Loof, L., Mendel-Hartvig, I., and Totterman, T.H. 1994. Primary biliary cirrhosis. High proportions of B cells in blood and liver tissue produce anti-mitochondrial antibodies of several Ig classes. J. Immunol. 153:2750-2757.

37. Falcone, M., Lee, J., Patstone, G., Yeung, B., and Sarvetnick, N. 1998. B lymphocytes are crucial antigen-presenting cells in the pathogenic autoimmune response to GAD65 antigen in nonobese diabetic mice. J. Immunol. 161:1163-1168.

38. Serreze, D.V., et al. 1998. B lymphocytes are critical antigen-presenting cells for the initiation of $\mathrm{T}$ cell-mediated autoimmune diabetes in nonobese diabetic mice. J. Immunol. 161:3912-3918. 